\title{
Electric Vehicle Routing Problem with Time Dependent Waiting Times at Recharging Stations
}

\author{
Merve Keskin \\ Faculty of Engineering and Natural Sciences, Sabanci University, Istanbul, Turkey \\ CIRRELT, Montréal, Canada \\ Gilbert Laporte \\ CIRRELT and Department of Management Sciences, HEC, Montréal, Canada \\ Bülent Çatay \\ Faculty of Engineering and Natural Sciences, Sabanci University, Istanbul, Turkey \\ Email: catay@sabanciuniv.edu
}

\section{Introduction and Problem Description}

The Electric Vehicle Routing Problem with Time Windows (EVRPTW) was introduced by [1] as an extension to the Green Vehicle Routing Problem of [2]. It is a variant of the Vehicle Routing Problem with Time Windows where a fleet of electric vehicles (EVs) is used instead of internal combustion engine vehicles. Since the EV has a limited driving range it may need to have its battery recharged at a station en route. In [1], it is assumed that the battery is fully recharged at any state of charge ( $\mathrm{SoC})$ and the duration is linearly proportional to the amount of energy transferred. The full recharge restriction was later relaxed in [3]. Furthermore, it is shown that the recharge duration is a concave function of the energy transferred and charging slows down when the energy level reaches $85 \%$ of the battery capacity [4-6].

The existing literature assumes that recharging at the station starts as soon as the EV arrives at the station. However, in practice the number of chargers in a station is limited and they may not be available at the time of arrival. So, the EV may have to queue before recharging and this waiting time may affect the routing decisions. The waiting time may vary depending on the location of the station and the time of the visit. Some variations in the waiting time are difficult to predict. For instance, if a charger is out of order, the EVs should queue in at another charger, if available, or go to a nearby station. On the other hand, some waiting time can be foreseen, for example when there are more vehicles on the roads during the rush hours, which translates in increased demand for recharging. In this study, we extend the EVRPTW by considering waiting times at the 
recharging stations. We assume an $\mathrm{M} / \mathrm{M} / 1$ queueing system and use expected waiting times to predict the queue lengths. We also use a nonlinear charging function and allow late arrivals at the customers and the depot with penalties. Moreover, we assume that the battery is operated between $10 \%$ and $90 \%$ of its capacity since it degrades faster beyond these limits [5]. Recently, [7] has addressed a similar environment where the stations have limited number of chargers (1,2 or 3 ) and an EV may need to wait for service if the chargers are busy recharging other EVs in the fleet. In this problem, the use of the chargers depends on the routing and charging decisions made, whereas in our setting the queue lengths are independent from our fleet, such as is the case at public stations.

In this context, we split the planning horizon (usually a day) into a predetermined number of time intervals such as morning, afternoon, evening, and night, and the average queue lengths at the stations vary according to the arrival time. We assume different EV arrival rates depending on the time of the day, e.g. the stations are less busy in the morning since the EVs depart with a full battery whereas the energy on the battery is usually consumed in the afternoon or evening, hence the EVs need recharging to continue their routes. The routing decisions are then made according to these time-dependent waiting times at the recharging stations. The objective is to minimize a total cost function which includes the energy cost, cost of vehicles, driver wages, and penalties associated with time window violations at the customers and the depot.

\section{Solution Methodology}

We assume that each recharging station is equipped with a single charger. The arrivals are Poisson with mean $\lambda$, and the service times follow an exponential distribution with parameter $\mu$. We apply a first come first served queue discipline where the first in first out property holds, i.e. the vehicles leave the station in the order they have arrived.

Our solution approach is a matheuristic which integrates the Adaptive Large Neighborhood Search (ALNS) method with an exact method. ALNS is a metaheuristic framework that iteratively destroys a solution and then repairs it using a greedy heuristic [8]. It has been successfully applied for solving various VRP variants in the recent literature. It uses several operators to destroy and repair the solution which are selected adaptively based on their past performances in improving the solution. In this study, we use the customer removal and insertion operators introduced in [3]. The repaired solution is accepted according to a simulated annealing criterion which allows the acceptance of worse solutions with a certain probability. Furthermore, we optimize the charging station selection and recharge quantity decisions of the vehicles by solving a mixed integer program using a commercial solver every after a predetermined number of iterations $\Delta$. Basically, the best solution obtained in the last $\Delta$ iterations is further enhanced by optimizing charging de- 
cisions while preserving the sequence of the customers. To solve this fixed-route problem fast, we formulate an effective mathematical model.

\section{Experimental Results}

To validate the performance of the proposed approach, we perform experiments using 36 small instances generated by [1]. We compare our results with those obtained with CPLEX. The service rate $\mu$ is calculated as explained in Section 1 . We assume $\lambda=\mu / 3$ for the least busy time interval and set the arrival rate $\lambda_{t}$ in time interval $t$ randomly by multiplying $\lambda$ with a positive scalar. All stations are assumed to have the same arrival rates for each time interval. Finally, the average waiting time at a station in time interval $t$ is calculated as $w_{t}=\lambda_{t} / \mu\left(\mu-\lambda_{t}\right) . \Delta$ is set to 200 and matheuristic terminates after 10,000 iterations. All experiments are conducted on an Intel Xeon E5 3.30 GHz processor and 32 GB of RAM. The mathematical model and the matheuristic are coded in Java and solved by CPLEX 12.6.2 with default settings.

Table 1: Results on Small Size Instances

\begin{tabular}{|c|c|c|c|c|c|c|c|c|c|}
\hline \multirow[b]{2}{*}{ Instance } & \multicolumn{2}{|c|}{ CPLEX } & \multicolumn{2}{|c|}{ Matheuristic } & \multirow[b]{2}{*}{ Instance } & \multicolumn{2}{|c|}{ CPLEX } & \multicolumn{2}{|c|}{ Matheuristic } \\
\hline & Cost & Time & Cost & Time & & Cost & Time & Cost & Time \\
\hline C101C10 & 3562.18 & 2233 & 3562.18 & 10 & C103C15 & 3589.52 & 7200 & 3583.61 & 26 \\
\hline C104C10 & 2348.00 & 7200 & 2347.63 & 31 & $\mathrm{C} 106 \mathrm{C} 15$ & 3476.10 & 7200 & 3476.10 & 30 \\
\hline C202C10 & 3904.82 & 7200 & 3904.82 & 38 & C202C15 & 4576.78 & 7200 & 3988.89 & 118 \\
\hline $\mathrm{C} 205 \mathrm{C} 10$ & 3417.17 & 160 & 3417.17 & 25 & C208C15 & 3753.73 & 7200 & 3753.73 & 125 \\
\hline $\mathrm{R} 102 \mathrm{C} 10$ & 1611.83 & 7200 & 1608.37 & 12 & $\mathrm{R} 102 \mathrm{C} 15$ & 2313.14 & 7200 & 2313.14 & 20 \\
\hline R103C10 & 1271.70 & 7200 & 1271.70 & 11 & R105C15 & 2185.12 & 7200 & 2185.05 & 16 \\
\hline R201C10 & 1289.27 & 6272 & 1289.27 & 48 & R202C15 & 1738.07 & 7200 & 1660.70 & 110 \\
\hline R203C10 & 1319.45 & 7200 & 1319.45 & 55 & R209C15 & 1494.19 & 7200 & 1487.39 & 130 \\
\hline $\mathrm{RC} 102 \mathrm{C} 10$ & 2211.27 & 7200 & 2211.27 & 8 & RC103C15 & 2342.45 & 7200 & 2342.45 & 10 \\
\hline $\mathrm{RC} 108 \mathrm{C} 10$ & 2073.69 & 7200 & 2073.69 & 9 & $\mathrm{RC} 108 \mathrm{C} 15$ & 2147.50 & 7200 & 2147.50 & 14 \\
\hline $\mathrm{RC} 201 \mathrm{C} 10$ & 1605.90 & 7200 & 1605.90 & 29 & RC202C15 & 1943.72 & 7200 & 1934.67 & 86 \\
\hline $\mathrm{RC} 205 \mathrm{C} 10$ & 1534.29 & 7200 & 1534.29 & 26 & RC204C15 & 1409.21 & 7200 & 1368.43 & 140 \\
\hline
\end{tabular}

The results are reported in Table 1 . The computation times are given in seconds. We set the time limit for CPLEX to 7200 seconds. So, if the computation time of CPLEX is less than this limit, the solution is optimal; otherwise, it is the best upper bound found. The matheuristic solutions are the best of 10 runs whereas the computational times are average of 10 runs. Since both CPLEX and our method found the optimal solution in all 5customer instances, we did not include them in the table. On average, the optimal solutions are attained in 206.8 and 10.7 seconds by CPLEX and the matheuristic, respectively. In 10-customer instances, the matheuristic provides better solutions in two instances which are highlighted in bold. In the remaining 10 instances, CPLEX and the matheuristic find the same solutions but CPLEX run times are significantly more and reach the time limit 
in most of the cases. In 15-customer instances the matheuristic achieves better solutions in 7 out of 12 instances. Furthermore, the run time is significantly better than CPLEX for all cases. Overall, we can claim that the proposed method outperforms CPLEX both in solution quality and computational time.

In conclusion, the proposed approach is effective in solving all small size instances. Our future work will concentrate on testing its performance on large instances under various scenarios of queue characteristics.

\section{References}

[1] M. Schneider, A. Stenger and G. Goeke, "The electric vehicle-routing problem with time windows and recharging stations", Transportation Science, 48(4), 500-520, 2014.

[2] S. Erdoğan, E. Miller-Hooks, "A green vehicle routing problem", Transportation Research Part E: Logistics and Transportation Review, 48(1), 100-114, 2012.

[3] M. Keskin, B. Çatay, "Partial recharge strategies for the electric vehicle routing problem with time windows", Transportation Research Part C: Emerging Technologies, 65: 111-127, 2016.

[4] T.M. Sweda, I.S. Dolinskaya and D. Klabjan, "Optimal recharging policies for electric vehicles", Transportation Science, 51(2), 457-479, 2017.

[5] S. Pelletier, O. Jabali, G. Laporte, M. Veneroni, "Battery degradation and behaviour for electric vehicles: Review and numerical analyses of several models", Transportation Research Part B: Methodological, 103, 158-187, 2017.

[6] A. Montoya, C. Guéret, J.E. Mendoza, J.G. Villegas, "The electric vehicle routing problem with nonlinear charging function", Transportation Research Part B: Methodological, 103, 87-110, 2017.

[7] A. Froger, J.E. Mendoza, O. Jabali, G. Laporte, "A Matheuristic for the Electric Vehicle Routing Problem with Capacitated Charging Stations", Technical Report 2017-31, CIRRELT, 2017.

[8] S. Ropke, D. Pisinger, D, "An adaptive large neighborhood search heuristic for the pickup and delivery problem with time windows", Transportation Science, 40(4), 455$472,2006$. 